\title{
Suppression of thyrotropin secretion during roxadustat treatment for renal anemia in a patient undergoing hemodialysis
}

\author{
Mitsuru Ichii ${ }^{1}$, Katsuhito Mori ${ }^{2}$, Daichi Miyaoka ${ }^{3}$, Mika Sonoda$^{1}$, Yoshihiro Tsujimoto ${ }^{1}$, Shinya Nakatani ${ }^{3}$,
} Tetsuo Shoji $i^{4}$ and Masanori Emoto ${ }^{2,3}$

\begin{abstract}
Background: Inhibition of hypoxia-inducible factor prolyl hydroxylase (HIF-PH) is a novel choice for the treatment of renal anemia, and an oral HIF-PH inhibitor roxadustat was approved for renal anemia. Roxadustat has high affinity to thyroid hormone receptor beta, which may affect thyroid hormone homeostasis.

Case presentation: We present here a patient undergoing hemodialysis with primary hypothyroidism receiving levothyroxine replacement, who showed decreased free thyroxine (FT4) and thyroid stimulating hormone (TSH) after starting roxadustat. Pituitary stimulation test revealed selective suppression of TSH secretion. Recovery of TSH and FT4 levels after stopping roxadustat suggested the suppression of TSH was reversible.
\end{abstract}

Conclusions: Physicians should pay special attention to thyroid hormone abnormalities in treatment with roxadustat.

Keywords: Renal anemia, Roxadustat, Thyroid stimulating hormone (TSH), Thyroid hormone receptor, Case report

\section{Background}

Renal anemia is an important complication which could affect quality of life of patients with chronic kidney disease (CKD) including those undergoing hemodialysis [1]. Anemia in CKD results from alterations of iron metabolism and impaired erythropoietin production by the kidneys $[2,3]$. The current standard treatment for anemia in patients on dialysis includes use of intravenous iron, use of erythropoiesis stimulating agents (ESA), and combination of them [2, 3]. Hypoxia-inducible factor (HIF) is involved in the impaired erythropoietin production in CKD [4] and rapidly degraded by HIF prolyl hydroxylase (HIF-PH). Thus, stabilization of HIF by HIF-PH inhibition is a novel strategy to renal anemia. Roxadustat, an oral inhibitor of HIF-PH, was shown be non-inferior to

\footnotetext{
* Correspondence: ktmori@med.osaka-cu.ac.jp

2Department of Nephrology, Osaka City University Graduate School of Medicine, 1-4-3, Asahi-machi, Abeno-ku, 545-8585 Osaka, Japan

Full list of author information is available at the end of the article
}

darbepoetin alfa [5]. In addition, its dose-effectiveness appears independent of serum C-reactive protein (CRP) levels, which may be advantage over ESA, because ESAresistance occurs in patients with high CRP levels [6].

Roxadustat has structure similar to triiodothyronine (T3) and also binds to thyroid hormone receptor $\beta$ $(T H R \beta)$ at a higher affinity than T3 [7]. Since THR $\beta$ in the pituitary and hypothalamus plays an important role in the feed-back regulation of thyroid hormone [8], roxadustat may affect the homeostasis of thyroid hormone.

Here, we describe a hemodialysis patient who showed suppression of thyroid stimulating hormone (TSH, or thyrotropin) and decreased free thyroxine (FT4) levels following roxadustat treatment for renal anemia.

\section{Case presentation}

A 77-year-old man with kidney failure due to undetermined etiology had been treated with hemodialysis for 7 years. He had history of surgery for esophageal cancer and

(c) The Author(s). 2021 Open Access This article is licensed under a Creative Commons Attribution 4.0 International License, which permits use, sharing, adaptation, distribution and reproduction in any medium or format, as long as you give appropriate credit to the original author(s) and the source, provide a link to the Creative Commons licence, and indicate if changes were made. The images or other third party material in this article are included in the article's Creative Commons licence, unless indicated otherwise in a credit line to the material. If material is not included in the article's Creative Commons licence and your intended use is not permitted by statutory regulation or exceeds the permitted use, you will need to obtain permission directly from the copyright holder. To view a copy of this licence, visit http://creativecommons.org/licenses/by/4.0/ The Creative Commons Public Domain Dedication waiver (http://creativecommons.org/publicdomain/zero/1.0/) applies to the data made available in this article, unless otherwise stated in a credit line to the data. 
gastric cancer before starting dialysis, and also hepatocellular carcinoma after starting dialysis. He was diagnosed at the age of 74 with subclinical primary hypothyroidism based on high level of TSH $(9.5 \mu \mathrm{IU} / \mathrm{mL})$ with normal level of FT4 $(1.0 \mathrm{ng} / \mathrm{dL})$. Hashimoto's thyroiditis was suspected based on clinical features including diffuse swelling of thyroid gland as well as hypoechoic and heterogenous pattern on thyroid ultrasonographic image, but autoantibodies such as anti-thyroid peroxidase and anti-thyroglobulin antibodies were negative. Since he complained of general fatigue and his laboratory test showed subclinical hypothyroidism, replacement therapy was started with $37.5 \mu \mathrm{g}$ per day of levothyroxine. In addition to levothyroxine, he was taking $5 \mathrm{mg}$ per day of cilnidipine and $40 \mathrm{mg}$ per day of telmisar$\tan$ for hypertension, $750 \mathrm{mg}$ per day of lanthanum carbonate for hyperphosphatemia, $1 \mathrm{mg}$ per day of evocalcet for secondary hyperparathyroidism, $0.5 \mathrm{mg}$ per day of entecavir hydrate for hepatitis B, $30 \mathrm{~mL}$ per day of lactulose syrup $60 \%$ for hyperammonemia, and 3 tables of a butyric acidproducing bacillus preparation for constipation.

Because his hemoglobin level did not improve despite treatment with $60 \mu \mathrm{g}$ of darbepoetin $\alpha$ once weekly and no sign of iron deficiency, treatment for anemia was switched to $100 \mathrm{mg}$ of roxadustat thrice weekly. After starting roxadustat, his hemoglobin level remained unchanged and he complained no specific symptom. However, decreased levels of both TSH and FT4 were noticed, as shown in Fig. 1, which was not attributable to known primary hypothyroidism.

Then, anterior pituitary function test was performed by combined intravenous administration of four hypothalamic releasing hormones. As shown in Table 1, the results revealed the selective and severe suppression of TSH response. Since the diagnose of central hypothyroidism was made in addition to the existing primary hypothyroidism, the dose of levothyroxine was increased from 37.5 to $62.5 \mu \mathrm{g}$ per day, and his FT4 was slightly increased. Magnetic resonance imaging did not show abnormal findings in the hypothalamus-pituitary region.

In search of possible signs of thyroid hormone deficiency, we reviewed routine laboratory measurements, vital signs, and body mass index. The results in Table 2 revealed remarkable reductions in serum total cholesterol, high-density lipoprotein cholesterol, and non-highdensity lipoprotein cholesterol levels. Monthly-averaged blood pressure and pulse rate levels measured at the start of hemodialysis session were slightly increased following roxadustat treatment. Serum creatinine level showed a reduction, whereas no appreciable changes were found in liver function tests or body mass index calculated from body weight at the end of hemodialysis session. These changes in laboratory tests and vital signs did not support thyroid hormone deficiency.

Because we suspected the possible influences of roxadustat, it was stopped, darbepoetin $\alpha$ was restarted, and the dose of levothyroxine was re-adjusted to $37.5 \mu \mathrm{g}$ per day. Then, his TSH was increased from 0.006 to 4.592 $\mu \mathrm{IU} / \mathrm{mL}$. His cholesterol, blood pressure, and pulse rate returned to the levels seen before roxadustat was started.

\section{Discussion and conclusion}

We report here a patient undergoing hemodialysis who showed decreased levels of TSH and FT4 levels after starting roxadustat treatment for renal anemia, in whom

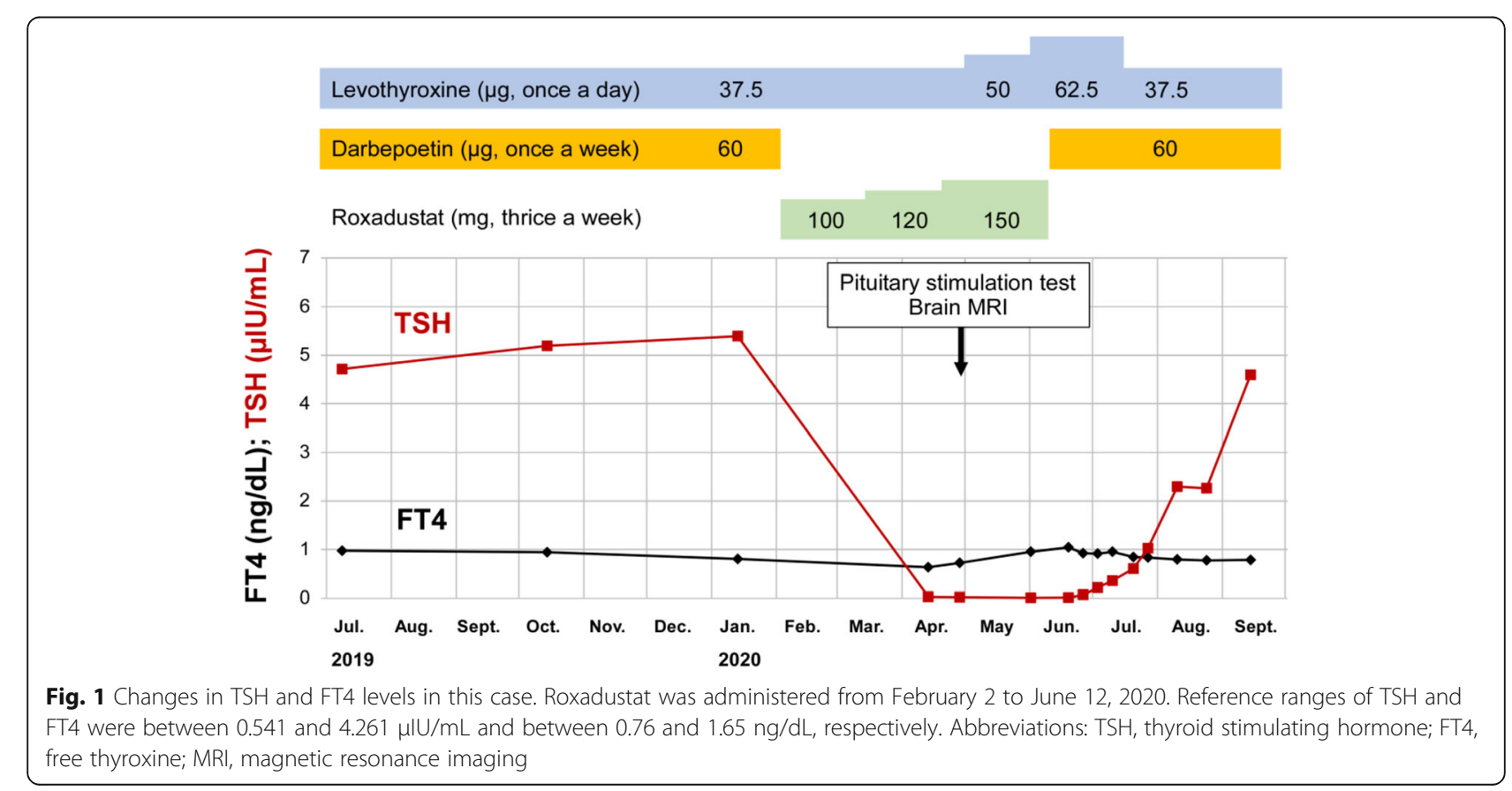


Table 1 Pituitary stimulation tests

\begin{tabular}{|c|c|c|c|c|c|c|}
\hline $\begin{array}{l}\text { Time } \\
\text { (minutes) }\end{array}$ & $\begin{array}{l}\text { TSH } \\
(\mu \mathrm{IU} / \mathrm{mL})\end{array}$ & $\begin{array}{l}\text { LH } \\
(\mu \mathrm{IU} / \mathrm{mL})\end{array}$ & $\begin{array}{l}\text { ACTH } \\
\text { (pg/mL) }\end{array}$ & $\begin{array}{l}\text { PRL } \\
\text { (ng/mL) }\end{array}$ & $\begin{array}{l}\text { COR } \\
(\mu \mathrm{g} / \mathrm{dL})\end{array}$ & $\begin{array}{l}\mathrm{GH} \\
(\mathrm{ng} / \mathrm{mL})\end{array}$ \\
\hline 0 & 0.017 & 87.7 & 44.7 & 17.4 & 6.1 & 2.2 \\
\hline 15 & 0.044 & 112.4 & 92.9 & 34.0 & 9.0 & 45.0 \\
\hline 30 & 0.054 & 123.2 & 93.6 & 34.6 & 10.0 & 61.3 \\
\hline 60 & 0.054 & 138.0 & 85.8 & 42.6 & 9.8 & 35.5 \\
\hline 90 & 0.062 & 145.9 & 70.4 & 39.7 & 9.0 & - \\
\hline 120 & 0.047 & 147.0 & 62.7 & 36.7 & 7.6 & - \\
\hline
\end{tabular}

The table gives the responses of the six anterior pituitary hormones to intravenous injection of the four hypothalamic releasing hormones ( $\mathrm{TRH}$, $\mathrm{LH}-\mathrm{RH}$, $\mathrm{CRH}$, and GH-RP2). Note that the TSH response was selectively suppressed.

Abbreviations: TSH thyroid stimulating hormone; $L H$ luteinizing hormone; $A C T H$ adrenocorticotropic hormone; PRL prolactin; COR cortisol; GH growth hormone; $T R H$ thyrotropin releasing hormone; $L H-R H$ luteinizing hormone releasing hormone; $C R H$ corticotropin releasing hormone; GH-RP2 growth hormone releasing protein 2

selective suppression of TSH from the anterior pituitary was demonstrated. The recovery of TSH after withdrawal of roxadustat suggests that the suppression of TSH is reversible.

Thyroid hormone is tightly controlled by the feedback regulation in the hypothalamus-pituitary-thyroid axis, and THRs play essential roles in the actions of thyroid hormone. THR and retinoid X receptor (RXR) form a heterodimer at the thyroid hormone responsive element of genes, and binding of T3 to THR promotes coactivator binding and gene transcription [9]. THR has isoforms with different tissue distributions accounting for tissue-specific action of thyroid hormone. THR $\beta$ plays an important role in hypothalamus and pituitary gland. According to Yao et al., roxadustat has a molecular structure similar to T3, and it binds to THR $\beta$ at a higher affinity than T3 [7]. In addition, THR $\beta$ has a predominant role in the down-regulation of thyrotropin releasing hormone (TRH) secretion from hypothalamus and TSH secretion from the anterior pituitary gland than THR $\alpha[8]$. Therefore, it is conceivable that roxadustat acts as an agonist of THR $\beta$, suppresses TSH secretion, and results in decreased serum FT4 level, as noticed in this case.

Perturbation of TSH by medication is known for other drugs. Bexarotene, used for treatment of cutaneous T-cell lymphoma, is known to cause central hypothyroidism [10]. Bexarotene has the structure of an RXR selective ligand. Binding of bexarotene with RXR promotes heterodimer complex formation with THR, inhibits secretion of $\mathrm{TSH}$, and central hypothyroidism in more than $90 \%$ of patients [11]. Nineteen of 27 patients noted that fatigue and cold intolerance, which were improved by levothyroxine [11]. Central hypothyroidism caused by bexarotene can be reversed by stopping the drug [10]. In contrast, nivolumab [12] and ipilimumab [13] can cause irreversible central hypothyroidism by inducing hypophysitis by an autoimmune mechanism.

The abnormal thyroid function tests observed in our patient were similar to the bexarotene-induced central hypothyroidism, but pathophysiologic implications may be different. Namely, the thyroid function tests in our patient do not necessarily indicate central hypothyroidism, because roxadustat could act as an agonist of THR $\beta$ not only in the hypothalamus and the pituitary gland, but also in peripheral tissues. Central hypothyroidism due to bexarotene was accompanied by hyperlipidemia [11] similar to elevated cholesterol and triglyceride levels often seen in primary hypothyroidism [14]. In contrast, the current case showed a reduction of serum cholesterol level after starting roxadustat, suggesting the excess rather than insufficiency of thyroid hormone function. In addition, the observed increase in blood pressure and pulse rate also supports this notion. Although reduced serum creatinine level is known for hyperthyroidism [15], the decreased serum creatinine in this case with kidney failure undergoing hemodialysis may be attributable not to increased kidney function but to alterations in skeletal muscles, which can be caused by the agonistic action to THR of roxadustat or by changes in thyroid hormone levels. Thus, we speculate the changes in thyroid hormone tests during roxadustat treatment indicate $\mathrm{T} 3$-mimetic action of roxadustat and excessive thyroid hormone functions in various tissues.

There are a few points which remain to be clarified. First, although decreased FT4 was treated by increasing the dose of levothyroxine in this case based on the diagnosis of central hypothyroidism in addition to the existing primary hypothyroidism, we are not sure that the diagonosis was correct. If roxadustat exerted its thyromimetic actions in this case, thyroid hormone replacement should have been reduced even when the level of FT4 was very low due to "pseudo" central hypothyroidism. Second, the cholesterol-lowering effect of roxadustat was known in the previous clinical trials in patients with anemia undergoing hemodialysis $[6,16]$, but its effects on thyroid hormone were not reported. Therefore, the possible link between thyroid hormone abnormalities and reduction in cholesterol following roxadustat may be worth exploring, but it is not straight-forward. Other possible explanations include reduced cholesterol 


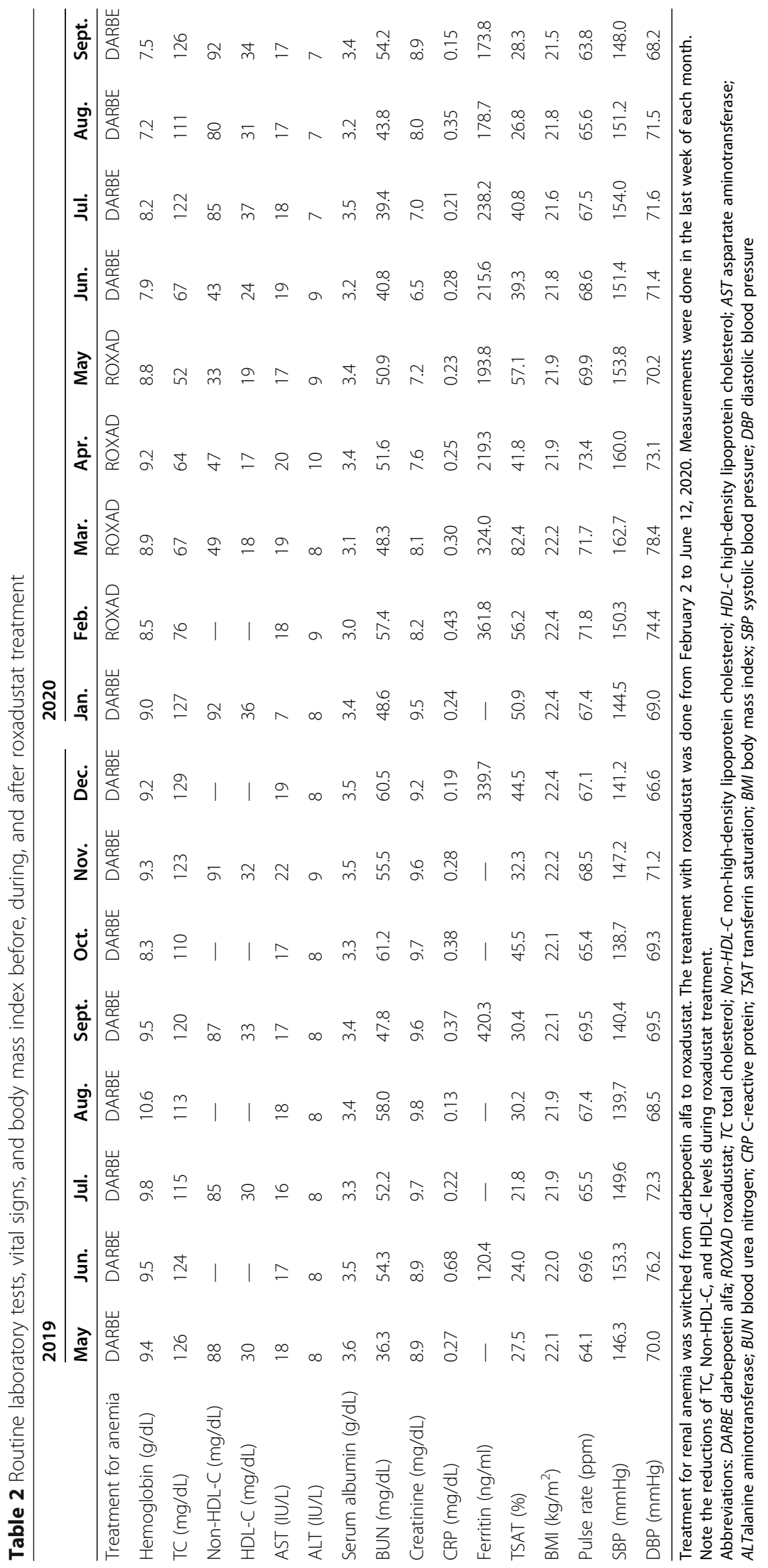


synthesis due to increased degradation of 3-hydroxy-3methylglutaryl-coenzyme A reductase stimulated by a mechanism mediated by HIF [17]. And third, since no adverse effect on thyroid hormone was reported in many patients in the clinical trials, the reason why this case was affected is unclear. The multiple comorbidities (including primary hypothyroidism, hepatitis, and cancer history) of this patient could be a factor which determined the susceptibility. Such patients with multiple comorbidities are likely to be excluded from clinical trials.

In conclusion, this is the first report of a case with renal anemia showing that treatment with roxadustat could interfere with the homeostasis of the hypothalamuspituitary-thyroid axis. Further information is needed for safe treatment of renal anemia with this novel agent.

\section{Abbreviations}

CKD: Chronic kidney disease; ESA: Erythropoiesis stimulating agents; HIF: Hypoxia-inducible factor; HIF-PH: HIF prolyl hydroxylase; CRP: C-reactive protein; T3: Triiodothyronine; THR: Thyroid hormone receptor; TSH: Thyroid stimulating hormone; FT4: Free thyroxine; RXR: Retinoid X receptor; TRH: Thyrotropin releasing hormone; MRI: Magnetic resonance imaging

\section{Acknowledgements}

The authors thank Yujiro Okute, Rino Nakaya, Shin Fukunaga, and Yuko Fujiwara (Division of Internal Medicine, Inoue Hospital) who were involved in clinical practice and provided considerable suggestion and discussion in this case report. Also, the authors thank the patient for granting permission to publish the case details as well as the staff of Inoue Hospital for their assistance.

\section{Authors' contributions}

Ml conceptualized this case report. MI, KM, DM, MS, YT, SN, TS, and ME contributed to acquisition and interpretation of data. TS and MI participated in writing the manuscript. MI, KM, DM, MS, YT, SN, TS, and ME have read and approved the final version for submission. MI, KM and TS substantially revised it.

\section{Funding}

None.

\section{Availability of data and materials}

Ml examined the patient. MI has full access to all data in this case report.

\section{Declarations}

Ethics approval and consent to participate

Not applicable.

\section{Consent for publication}

Written informed consent for publication was obtained from the present patient. A copy of the written consent is available for review by the Editor of this journal.

\section{Competing interests}

$\mathrm{Ml} \mathrm{received} \mathrm{an} \mathrm{honorarium} \mathrm{for} \mathrm{lecturing} \mathrm{from} \mathrm{Astellas} \mathrm{Pharma} \mathrm{Inc.} \mathrm{and}$ Kyowa Kirin Co., Ltd. KM received an honorarium for lecturing from Astellas Pharma Inc., Mitsubishi Tanabe Pharma Corporation and Kyowa Kirin Co., Ltd. YT received an honorarium for lecturing from Astellas Pharma Inc. and Kyowa Kirin Co., Ltd. SN received an honorarium for lecturing from Kyowa Kirin Co., Ltd. ME received an honorarium for lecturing from Astellas Pharma Inc., Mitsubishi Tanabe Pharma Corporation and Kyowa Kirin Co., Ltd. DM, MS, and TS have no conflict of interest.

\section{Author details}

'Division of Internal Medicine, Inoue Hospital, Suita, Japan. ${ }^{2}$ Department of Nephrology, Osaka City University Graduate School of Medicine, 1-4-3, Asahi-machi, Abeno-ku, 545-8585 Osaka, Japan. ${ }^{3}$ Department of Metabolism Endocrinology and Molecular Medicine, Osaka City University Graduate
School of Medicine, Osaka, Japan. ${ }^{4}$ Department of Vascular Medicine, Osaka City University Graduate School of Medicine, Osaka, Japan.

Received: 30 August 2020 Accepted: 10 March 2021

Published online: 20 March 2021

References

1. Moreno F, Aracil FJ, Perez R, Valderrabano F. Controlled study on the improvement of quality of life in elderly hemodialysis patients after correcting end-stage renal disease-related anemia with erythropoietin. Am J Kidney Dis. 1996:27(4):548-56.

2. Drueke TB, Parfrey PS. Summary of the KDIGO guideline on anemia and comment: reading between the (guide)line(s). Kidney Int. 2012;82(9):952-60.

3. Yamamoto H, Nishi S, Tomo T, Masakane I, Saito K, Nangaku M, Hattori M, Suzuki T, Morita S, Ashida A, et al. 2015 Japanese Society for Dialysis Therapy: Guidelines for Renal Anemia in Chronic Kidney Disease. Renal Replacement Therapy. 2017;3(1):36

4. Gupta N, Wish JB. Hypoxia-Inducible Factor Prolyl Hydroxylase Inhibitors: A Potential New Treatment for Anemia in Patients With CKD. Am J Kidney Dis. 2017;69(6):815-26

5. Akizawa T, Iwasaki M, Yamaguchi Y, Majikawa Y, Reusch M. Phase 3, Randomized, Double-Blind, Active-Comparator (Darbepoetin Alfa) Study of Oral Roxadustat in CKD Patients with Anemia on Hemodialysis in Japan. J Am Soc Nephrol. 2020;31(7):1628-39.

6. Provenzano R, Besarab A, Wright S, Dua S, Zeig S, Nguyen P, Poole L, Saikali KG, Saha G, Hemmerich S, et al: Roxadustat (FG-4592) Versus Epoetin Alfa for Anemia in Patients Receiving Maintenance Hemodialysis: A Phase 2, Randomized, 6- to 19-Week, Open-Label, Active-Comparator, Dose-Ranging, Safety and Exploratory Efficacy Study. Am J Kidney Dis 2016, 67(6):912-924.

7. Yao B, Wei Y, Zhang S, Tian S, Xu S, Wang R, Zheng W, Li Y. Revealing a Mutant-Induced Receptor Allosteric Mechanism for the Thyroid Hormone Resistance. iScience. 2019;20:489-96.

8. Koulouri O, Moran C, Halsall D, Chatterjee K, Gurnell M. Pitfalls in the measurement and interpretation of thyroid function tests. Best Pract Res Clin Endocrinol Metab. 2013;27(6):745-62.

9. Brent GA. Mechanisms of thyroid hormone action. J Clin Invest. 2012;122(9): 3035-43.

10. Sherman SI, Gopal J, Haugen BR, Chiu AC, Whaley K, Nowlakha P, Duvic M. Central hypothyroidism associated with retinoid $X$ receptor-selective ligands. N Engl J Med. 1999;340(14):1075-9.

11. Hamada T, Tokura $Y$, Sugaya M, Ohtsuka M, Tsuboi R, Nagatani T, Kiyohara $E$, Tani M, Setoyama M, Matsushita S, et al. Long-term efficacy and safety of bexarotene for Japanese patients with cutaneous T-cell lymphoma: The results of a phase 2 study (B-1201). J Dermatol. 2019:46(7):557-63.

12. Alhusseini M, Samantray J. Hypothyroidism in Cancer Patients on Immune Checkpoint Inhibitors with anti-PD1 Agents: Insights on Underlying Mechanisms. Exp Clin Endocrinol Diabetes. 2017;125(4):267-9.

13. Haissagerre M, Prey S, Lauro C, Rousset M, Georges A, Corcuff JB. Immunotherapy-induced hypothyroidism A report of melanoma treated by ipilimumab and nivolumab. Ann Biol Clin (Paris). 2018;76(3):326-8.

14. Duntas LH, Brenta G. A Renewed Focus on the Association Between Thyroid Hormones and Lipid Metabolism. Front Endocrinol (Lausanne). 2018:9:511.

15. Mariani LH, Berns JS. The renal manifestations of thyroid disease. J Am Soc Nephrol. 2012;23(1):22-6.

16. Chen N, Hao C, Liu BC, Lin H, Wang C, Xing C, Liang X, Jiang G, Liu Z, Li X, et al. Roxadustat Treatment for Anemia in Patients Undergoing Long-Term Dialysis. N Engl J Med. 2019;381(11):1011-22.

17. Nguyen AD, McDonald JG, Bruick RK, DeBose-Boyd RA. Hypoxia stimulates degradation of 3-hydroxy-3-methylglutaryl-coenzyme A reductase through accumulation of lanosterol and hypoxia-inducible factor-mediated induction of insigs. J Biol Chem. 2007:282(37):27436-46.

\section{Publisher's Note}

Springer Nature remains neutral with regard to jurisdictional claims in published maps and institutional affiliations. 